\title{
Correction to: An Exploratory Study of Hyoid Visibility, Position, and Swallowing-Related Displacement in a Pediatric Population
}

\author{
Aimee Riley $^{1} \cdot$ Anna Miles $^{1,4}$ (D) $\cdot$ Catriona M. Steele ${ }^{2,3}$ \\ Published online: 10 September 2020 \\ (c) Springer Science+Business Media, LLC, part of Springer Nature 2020
}

\section{Correction to: Dysphagia (2019) 34:248-256 https://doi.org/10.1007/s00455-018-9942-3}

We are writing to inform the readers of the Dysphagia journal of an error in the following paper: Riley A, Miles A \& Steele CM. An Exploratory Study of Hyoid Visibility, Position, and Swallowing-Related Displacement in a Pediatric Population. Dysphagia. 2019, 34 (2):248-256.

The aim of this study was to investigate the feasibility of visualizing and measuring position and swallowing-related displacement of the hyoid bone in children. Videofluoroscopies from a clinical dataset comprising recordings from 133 children aged 9 days to 21 years were analyzed. The hyoid bone was not visible in children under 9 months of age.

When visible, minimum hyoid position was measured on frames showing the pharynx at rest and maximum hyoid position was measured along the anterior $(\mathrm{X})$, superior $(\mathrm{Y})$ and hypotenuse $(\mathrm{XY})$ axes, relative to the anterior-inferior corner of the $\mathrm{C} 4$ vertebra on frames showing the hyoid at its peak anterior-superior position. An open-source spreadsheet previously available from the Steele Swallowing Lab (https://steeleswallowinglab.ca) was used to calculate hyoid position. Additionally, measures of displacement (from rest to peak) were calculated along each axis. Results were

The original article can be found online at https://doi.org/10.1007/ s00455-018-9942-3.

Anna Miles

a.miles@auckland.ac.nz

Speech Science, The University of Auckland, Private Bag 92019, Auckland 1142, New Zealand

2 Toronto Rehabilitation Institute - University Health Network, Toronto, ON, Canada

3 Rehabilitation Sciences Institute, University of Toronto, Toronto, ON, Canada

4 Speech Science, School of Psychology, The University of Auckland, Private Bag 92019, Auckland 1142, New Zealand compared to previously reported reference data for healthy adults using forest plots [1].

It has subsequently come to our attention that an error in the mathematical formula in the spreadsheet for calculating hyoid position resulted in a reversal of the $\mathrm{X}$ and $\mathrm{Y}$ planes of measurement. For this paper, the point measures of minimum and peak hyoid position were affected by the formula error in the calculation spreadsheet, such that the anterior $(\mathrm{X})$ and superior $(\mathrm{Y})$ results were transposed. None of the other reported measures were affected. Corrections to relevant tables and figures are provided in Table 1 and Fig. 1. Italic rows represent corrected data.

The impact of these corrections on the interpretation of data in the manuscript is as follows.

It was previously reported that hyoid position values at rest in children fell above the upper 95\% confidence interval boundaries for adults along the $\mathrm{Y}$ and $\mathrm{XY}$ axes. The correction confirms that this was also true along the $\mathrm{X}$ axis, showing that the hyoid is positioned both higher and further forward at rest in children. Previously reported differences in peak hyoid position between children and adults, in the form of a higher Y position, were no longer observed after correction of the data. Rather, peak position measures in the children overlapped with the $95 \%$ confidence intervals for adults along all 3 axes of measurement.

We trust that these corrections will clarify any misunderstandings that may have occurred based on the originally reported results for these three studies.

\section{Reference}

1. Molfenter SM, Steele CM. Kinematic and temporal factors associated with penetration-aspiration in swallowing liquids. Dysphagia. 2014;29(2):269-76. 
Table 1 (Correction to Original Table 2) Descriptive statistics for all nine hyoid parameters with effects of age (covariate) and Penetration-Aspiration Scale (PAS) scores

\begin{tabular}{|c|c|c|c|c|c|c|}
\hline \multirow[t]{2}{*}{ Parameter } & \multicolumn{2}{|c|}{ Descriptives } & \multicolumn{2}{|l|}{ Age } & \multicolumn{2}{|c|}{$\mathrm{PAS}<3$ vs $\geq 3$} \\
\hline & Mean & $95 \% \mathrm{CI}$ & $F$ value & $p$ value & $F$ value & $p$ value \\
\hline Minimum XY position (\%C2-4) & 123.88 & $112.14-135.61$ & 5.91 & 0.02 & 2.51 & 0.12 \\
\hline Minimum X position (\%C2-4) & 138.78 & $129.50-148.06$ & 23.47 & $<0.001$ & 1.21 & 0.28 \\
\hline Minimum Y position (\%C2-4) & 30.8 & $16.76-44.85$ & 0.351 & 0.56 & 1.66 & 0.21 \\
\hline Peak XY position (\%C2-4) & 195.98 & $184.25-207.71$ & 17.86 & $<0.001$ & 2.42 & 0.13 \\
\hline Peak X position (\%C2-4) & 167.91 & $158.67-177.16$ & 18.64 & $<0.001$ & 0.77 & 0.39 \\
\hline Peak Y position (\%C2-4) & 93.26 & $78.05-108.47$ & 1.61 & 0.21 & 1.28 & 0.26 \\
\hline $\mathrm{XY}$ displacement $(\% \mathrm{C} 2-4)$ & 72.1 & $60.95-83.25$ & 3.564 & 0.07 & 0.001 & 0.98 \\
\hline Anterior displacement (\%C2-4) & 28.77 & $29.86-34.72$ & 5.78 & $<0.05$ & 0.01 & 0.94 \\
\hline Superior displacement $(\% \mathrm{C} 2-4)$ & 60.02 & $61.70-72.55$ & 0.88 & 0.35 & 0.15 & 0.7 \\
\hline
\end{tabular}

$\% \mathrm{C} 2-4=\%$ of the distance of the second to fourth cervical vertebrae. Italic rows are corrected values
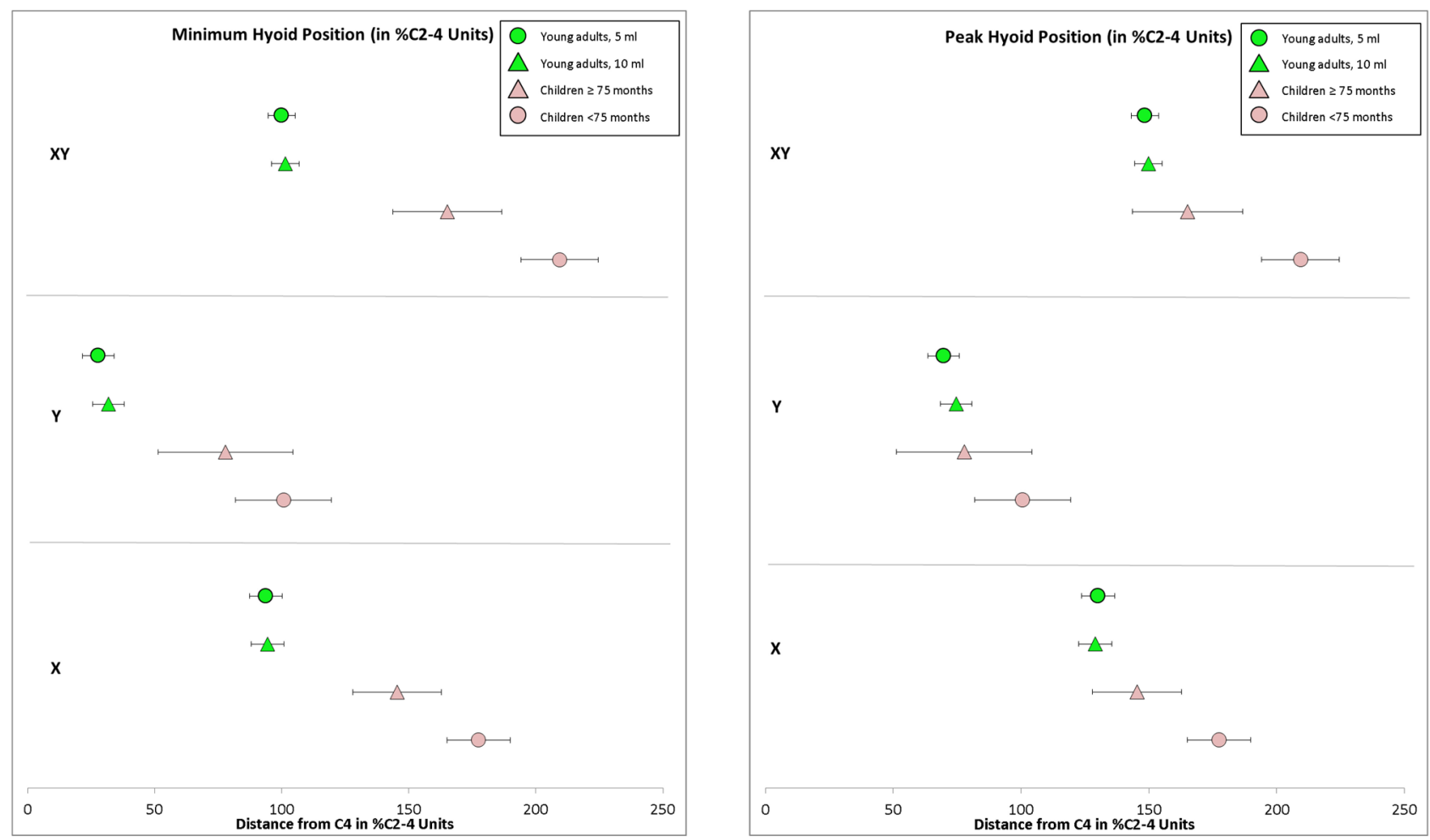

Fig. 1 (Correction to Original Fig. 4) Forest plots comparing hyoid position measures for children with adult norms [1]

Publisher's Note Springer Nature remains neutral with regard to jurisdictional claims in published maps and institutional affiliations. 